\title{
A Systemic Approach to Pediatric Chronic Health Conditions: Why We Need to Address Parental Stress
}

\author{
Lisa-Marie Emerson $^{1} \cdot$ Susan Bögels ${ }^{2}$
}

Published online: 13 July 2017

(C) Springer Science+Business Media, LLC 2017

Chronic health conditions in childhood are on the rise (Van Cleave et al. 2010). Associated psychological distress is not uncommon. The child does not experience these difficulties in isolation, but rather sits at the center of a network of systems; the most pertinent of which is the family. Childhood distress in relation to the chronic condition has an intimate bidirectional relationship with family functioning; caregiver distress is a key contributing factor to child outcomes.

Parental suffering in relation to pediatric chronic conditions is likely to occur at three main time points. First, prior to the diagnosis, the family is likely to have struggled with managing the child's ill health, and experienced disruption to idealized family life. Second, parents will experience a reaction to the diagnosis of the condition, which may include complex feelings of loss, guilt, fear and even shame. Third, parents will experience a period of adjustment, including challenges of condition management and supporting their child longer-term. It is unsurprising that high stress and burnout are consistently reported by parents of children with chronic conditions (Cousino and Hazen 2013). Parent distress predicts the child's psychological adjustment and reduces the parent's ability to effectively manage the condition (e.g. reduced vigilance and adherence) (Wood et al. 2015).

Lisa-Marie Emerson

1.emerson@sheffield.ac.uk

1 Clinical Psychology Unit, Department of Psychology, University of Sheffield, Cathedral Court, 1 Vicar Lane, Sheffield S1 2LT, UK

2 Research Institute of Child Development and Education, University of Amsterdam, Amsterdam, The Netherlands
Wherever possible, the primary intervention for chronic conditions is necessarily medical, and directed at managing the condition. Psychological support commonly focuses on specialist input for children who experience severe adjustment problems (e.g. behavioral or emotional difficulties), and neglects the distress experienced by many parents. Parent distress interferes with their ability to assimilate new information at the point of diagnosis; subsequently, parents report a lack of information or misinformation and distrust for healthcare professionals (Kepreotes et al. 2010). The risk is an increased chance of failure in condition management. The resultant follow-up from healthcare professionals is likely to contribute to further distress, as parents perceive themselves to have failed. This vicious cycle poses the risk of secondary child and parental mental health problems, disrupted parent-child relationship and family functioning, which may negatively impact the child's primary somatic condition.

The role of the family system has long been acknowledged in pediatric psychiatric settings as a contributing factor to the maintenance of child distress and a point for intervention. In neurodevelopmental conditions, such as autism and Attention Deficit Hyperactivity Disorder (ADHD), parents are generally not seen to have contributed to the cause of the condition, but rather parental reaction is pivotal in condition management. Parent stress may cause parenting styles that maintain difficulties of adjustment. For example, over-reactive parenting can exacerbate restlessness and impulsivity in a child with ADHD, or overprotective parenting can maintain dependency in a child with autism. Psychological support for parents is a key part of managing pediatric neurodevelopmental conditions and often compliments medication, with positive outcomes. For example, it can be a prerequisite of receiving a diagnosis or 
medication for ADHD that parents also attend specialist training in managing child behavior (e.g. UK clinical guidelines for ADHD). This combined treatment approach supports necessary systemic changes and gives parents the opportunity to explore the interaction of the condition with family life. Interventions that target parents' own stress related to the child's condition, such as mindful parenting groups, reduce parental stress and related psychopathology, and improve the child's condition.

We propose a similar model for understanding the contributing role of parents in pediatric chronic health conditions, whereby parents may not be a causal factor in the diagnosis, but play a contributing role to child outcomes. A combined healthcare approach (medical plus systemic support) that acknowledges the role of parent distress could lead to improvements in child outcomes via three indirect routes. First, parents will be in a better position to help manage their child's condition (e.g. medication compliance) because they will be able to assimilate new information and act on it. Second, acknowledging and supporting parents in their adjustment to their child's condition will enable parents to model to their child a healthy response to the new challenges. Third, parents will have increased confidence in the medical professionals, which is pivotal in enabling parents to adjust to the new task of supporting a child with a chronic condition.

To date psychological interventions that include parents have failed to directly target parental stress, and so have not reduced parental problems. An exception is problemsolving therapy, which reduces parental stress and psychopathology (Eccleston et al. 2015). We propose that interventions that target intrapsychic processes at the heart of parents' suffering will be most effective. One such novel approach is mindful parenting, which aims to reduce parental stress by increasing acceptance and self-care. As a parenting style, mindful parenting is associated with better diabetes management $\left(\mathrm{HbA}_{1 \mathrm{c}}\right.$ and hospitalization) and quality of life in adolescents (Serkel-Schrama et al. 2016). As an intervention, Mindful Parenting is effective for neurodevelopmental conditions, but has not yet been tested for pediatric chronic health conditions. We predict that such an approach would substantially reduce parental stress, and have knock-on effects on child outcomes.

A healthcare approach for pediatric chronic conditions has implications for research and clinical service planning. Research and service-user involvement should ascertain what support parents could benefit from. Fathers have not been included in many parenting interventions and their role should be addressed. Relatedly, the role of co-parenting in managing a child's condition should be investigated. Pediatric services for chronic conditions could provide integrated support to parents to manage their own suffering. Parent groups may offer the most cost-effective approach; such interventions can be scaled up (e.g., mindful parenting to groups of around 20 parents), and promote parental acceptance, prevent isolation, and fulfill families' need for support in a larger community, thereby also reducing dependency on the healthcare system. Although the beneficial effects of parental support are self-evident, providing such services may not be easy. For example, the US healthcare system, which is based on the market justice principle, adversely impacts low-income parents whose children have chronic conditions. However, this should not preclude us from developing the evidence base on which future social justice healthcare systems can be built.

\section{Compliance with Ethical Standards}

Conflict of Interest The authors declare that they have no competing interests.

Ethical Approval This article does not contain any studies with human participants performed by any of the authors.

\section{References}

Cousino, M. K., \& Hazen, R. A. (2013). Parenting stress among caregivers of children with chronic illness: A systematic review. Journal of Pediatric Psychology, 38, 809-828.

Eccleston, C., Fisher, E., Law, E., Bartlett, J., \& Palermo, T. M. (2015). Psychological interventions for parents of children and adolescents with chronic illness (Review). Cochrane Database Systematic Reviews, 15(4), CD009660.

Kepreotes, E., Keatinge, D., \& Stone, T. (2010). The experience of parenting children with chronic health conditions: A new reality. Journal of Nursing and Healthcare of Chronic Illness, 2, 51-62.

Serkel-Schrama, I. J. P., de Vries, J., Nieuwesteeg, A. M., Pouwer, F., Nyklicek, I., \& Speight, J., et al. (2016). The association of mindful parenting with glycemic control and quality of life in adolescents with type 1 diabetes: Results from diabetes MILES the Netherlands. Mindfulness, 7, 1227-1237.

Van Cleave, J., Gortmaker, S. L., \& Perrin, J. M. (2010). Dynamics of obesity and chronic health conditions among children and youth. JAMA, 303, 623-630.

Wood, B. L., Miller, B. D., \& Lehman, H. K. (2015). Review of family relational stress and pediatric asthma: The value of biopsychosocial systemic models. Family Process, 54, 376-389. 